\title{
Analytical STEM/EDS Characterization of Elemental Segregation and Solid Solution Formation in Multiphase Ceramics
}

\author{
Komal Syed ${ }^{1}$, Mingjie Xu ${ }^{1,2}$, David Kok ${ }^{1}$, Kenta K. Ohtaki ${ }^{1}$ and Martha L. Mecartney ${ }^{1}$ \\ 1. Department of Materials Science \& Engineering, University of California, Irvine, CA, USA. \\ 2. Irvine Materials Research Institute (IMRI), University of California, Irvine, CA, USA.
}

Bulk ceramic processing includes the densification of powders at high temperature. This study evaluates elemental segregation at grain boundaries and solid solution formation for a three-phase ceramic $\mathrm{Al}_{2} \mathrm{O}_{3}-$ $\mathrm{MgAl}_{2} \mathrm{O}_{4}-8$ mole $\% \mathrm{Y}_{2} \mathrm{O}_{3}$ stabilized cubic $\mathrm{ZrO}_{2}$ (YSZ) made under different processing conditions: (1) conventional sintering, (2) two step-sintering, (3) spark plasma sintering (SPS), and (4) flash sintering ${ }^{[1,2]}$. In addition, the stability of interfaces and phases under $946 \mathrm{MeV} \mathrm{Au}$ ion irradiation is analyzed. Segregation at grain boundaries and point defect generation in the bulk can serve to disrupt phonon propagation, reducing thermal conductivity.

TEM samples were prepared by using a TESCAN GAIA3 FIB-SEM. JEOL 2800 and JEOL Grand ARM300CF TEM/STEM at 300keV, both equipped with dual silicon drift detectors for energy dispersive spectroscopy (EDS), were used to characterize the grain boundaries and to understand elemental segregation. Both EDS spectral maps and line scans were obtained for analysis. The electron beam probe size was $0.1 \mathrm{~nm}$ on the Grand ARM and $1 \mathrm{~nm}$ on the JEOL 2800. The EDS parameters were optimized based on minimizing beam damage versus increasing net EDS counts.

There are three phases, YSZ, alumina, spinel, in this material and six types of grain boundaries (Fig.1, conventionally sintered). $\mathrm{Mg}$ and $\mathrm{Al}$ are found to be present in varying amounts in solid solution in YSZ and $\mathrm{Al}$ is highly segregated at $Y S Z / Y S Z$ grain boundaries, with a depletion of Zr (Fig. 2, flash sample). In Spinel/Spinel grain boundaries, there is depletion of $\mathrm{Mg}$ at the grain boundary accompanied by the segregation of $\mathrm{Y}$ and $\mathrm{Zr}$ (Fig.3, SPS). Similar observations are made for Alumina/Alumina grain boundaries and Spinel/Alumina grain boundaries. In addition, there is a variation of the $\mathrm{Mg} / \mathrm{Al}$ ratio in the $\mathrm{MgAl}_{2} \mathrm{O}_{4}$ grains, depending on the sintering process. Higher temperatures reached during processing expand the solution solubility of $\mathrm{Al}_{2} \mathrm{O}_{3}$ in $\mathrm{MgAl}_{2} \mathrm{O}_{4}$. YSZ/Spinel and YSZ/Alumina interfaces do not have a significant amount of elemental segregation which is not surprising as these boundaries are heterogeneous with different cations on each side. However, these interfaces in the SPS samples were not as resistant to electron beam damage compared to the conventionally sintered samples. The higher stability in conventionally sintered samples may be due to the structure of the boundaries with high temperature, long anneal times in contrast to the rapid sintering at lower temperatures achieved in SPS resulting in higher energy non-equilibrium grain boundaries.

In SPS samples, $\mathrm{YSZ}_{\mathrm{MgAl}} \mathrm{O}_{4}$ interfaces show depletion of $\mathrm{Mg}$ and $\mathrm{O}$ in the spinel grain near the boundary but enrichment of $\mathrm{Mg}$ at the interface boundary. Al depletion is also observed at $\mathrm{YSZ} / \mathrm{Al}_{2} \mathrm{O}_{3}$ boundaries to some extent. However, $\mathrm{Al}$ depletion is not as prominent as the loss of $\mathrm{Mg}$ and $\mathrm{O}$ and is not observed for most boundaries analyzed. The stoichiometric loss of $\mathrm{Mg}$ and $\mathrm{O}$ is believed to be due to gradual volatilization in the vacuum of TEM ${ }^{[3]}$. Irradiated SPS samples were found to be even more prone to ebeam damage, particularly at $\mathrm{YSZ}_{\mathrm{MgAl}} \mathrm{O}_{4}$ grain boundaries (Fig.4, SPS irradiated). The elemental segregation and inhomogeneity observed would all contribute to lower thermal conductivity [4]. 
References:

[1] JP Kelly and OA Graeve, JOM 67 (2015), p. 29.

[2] M Cologna, ALG Prette and R Raj, J Am Ceram Soc 94 (2011), p. 316.

[3] P Carrez, H Leroux and P Cordier, Philos Mag A 81 (2001), p. 2823.

[4] This work is supported by NSF DMR Thermal Grant 442660-21130.

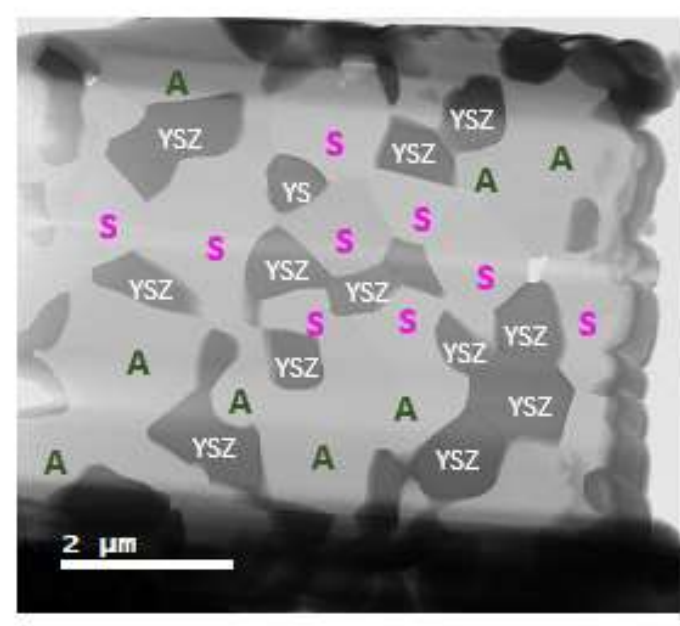

Fig. 1: BF STEM image of 3-phase conventionally sintered.
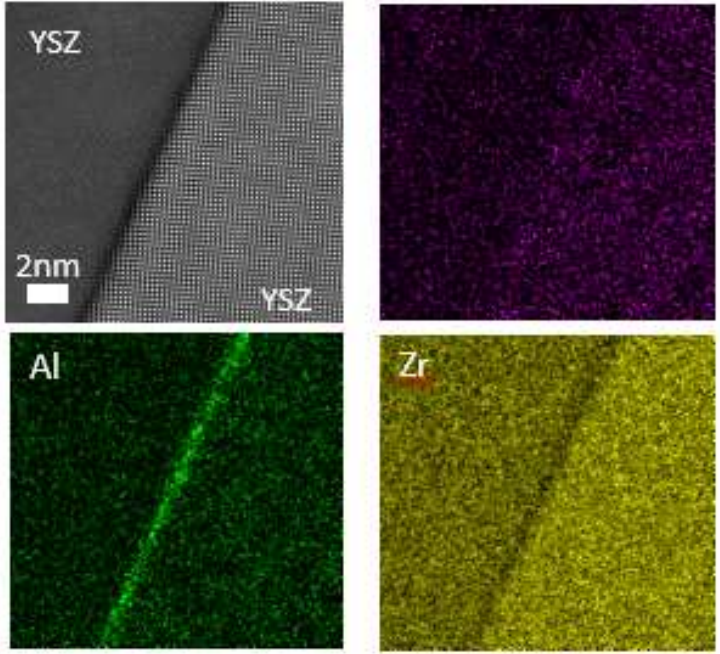

Fig. 2: DF STEM and EDS maps of YSZ/YSZ boundary with Al segregation in flash sintered sample.
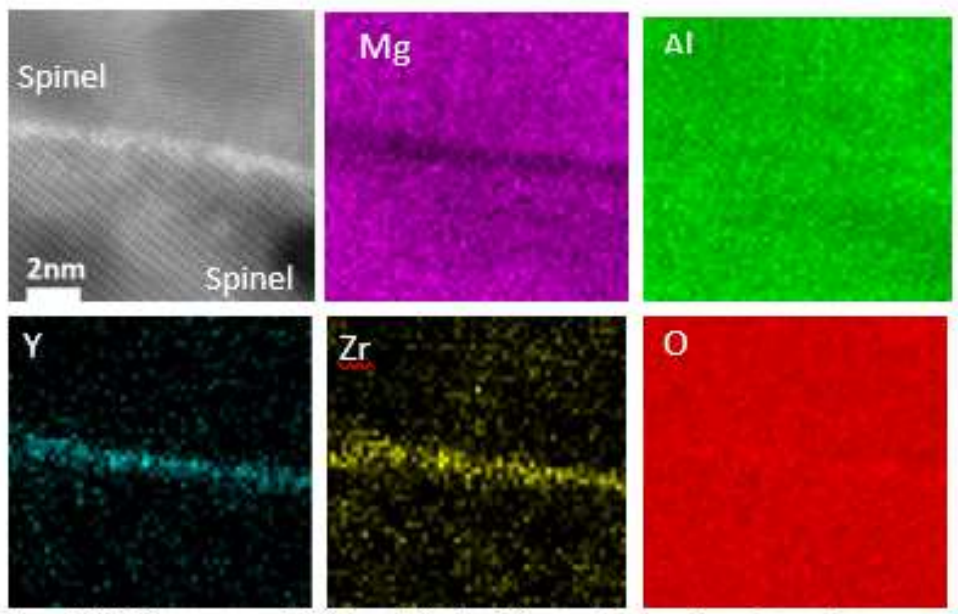

Fig. 3: DF STEM and EDS maps of Spinel/Spinel boundary showing elemental segregation in SPS nonirradiated sample.

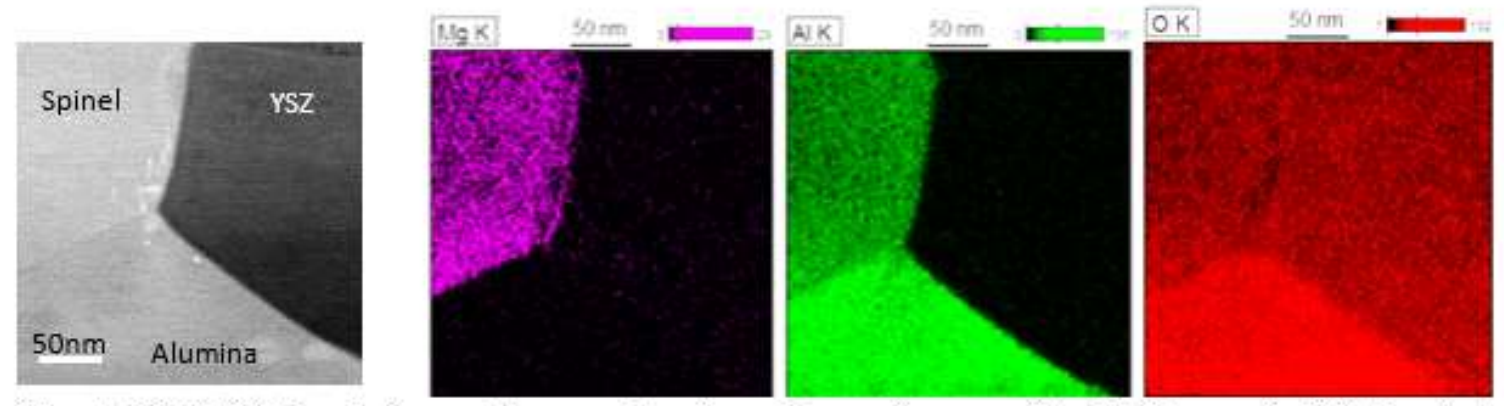

Fig. 4: BF STEM and elemental maps showing e-beam damage after EDS scan in SPS irradiated sample. 